\title{
On the factorization of lacunary polynomials
}

\author{
H.W. Lenstra, Jr.
}

\section{To Andrzej Schrnzel}

\begin{abstract}
Descartes's rule of signs implies that the number of non-vamishing real zeroes of a non-zero polynomial $f$ in one variable with real coefficients is at most $2 k$, if $k+1$ is the number of non-zero terms of $f$ In this paper the following non-archimedean analogue is obtaned Let $p$ be a prime number, $L$ a field that is a finite extension of the field of $p$-adic numbers, and $k$ a positive integer $T$ hen there exists a positive integer $B=B(h, L)$ with the following property if $f \in L[X]$ has at most $k+1$ non-zero terms, and $f \neq 0$, then $f$ has at most $B$ non vanishing zcroes in $L$, counting multiplicities For cxample if $L$ is the field of 2 adic numbers, and $k=2$, then one can take $B=6$ As a consequence, it is shown that for any three positive integers $m, k$, and $d$ there exists a positive integer $A=A(m, k, d)$ with the following property Suppose that $K$ is an algebraic number field of degree at most $m$ over the field of rational numbers, that $f \in K[X]$ is a non-zero polynomial with at most $k+1$ non-zero terms, and that $g \in K[X]$ is a factor of $f$ such that each irreducible factor of $g$ has degree at most $d$ and such that $g(0) \neq 0$ Then the degree of $g$ is at most $A$ The value for $A$ given by the proof satisfies $A(m, h, d)=O\left(k^{2} 2^{m d} m d \log (2 m d k)\right)$, the $O$-constant being absolute and effectively computable
\end{abstract}

1991 Mathematıcs Subject Classific atıon Prımary 11R09, 11 S05

Key words lacunary polynomial, $p$-adıc numkers Descartes's rule of signs

Acknowledgements. The author was supp orted by NSF under grant No DMS 92-24205 He thanks J A Csirık M Filasetd, B Poonen, A Schinzel, R Tudeman, and J D Vaaleı for helpful advice

\section{1. introduction}

Let $\mathbf{Q}$ denote the field of rational numbers, and for a ring $R$, wilte $R[X]$ for the ring of polynomials in onc variable $X$ over $R$

Theorem 1. For any three positive integers $m, k$, and $d$ there exusts a positive integer $A=A(m, k, d)$ with the followng property Suppose that $K$ us an algebrauc number ficld of degree at most in over $\mathrm{Q}$, that $f \in K[X]$ is a non zero polynomial 
with at most $k+1$ non-zero terms, and that $g \in K[X]$ is a factor of $f$ such that each urreducuble factor of $g$ has degree at most $d$ and such that $g(0) \neq 0$ Then the degree of $g$ us at most $A$

Note that the bound $A$ is independent of the cocfficients and the degree of $f$

With $d=1$, the theorem implies a bound $A=A\left(\left[\begin{array}{ll}K & \mathbf{Q}\end{array}\right], k, 1\right)$ on the number of non-vanushing zeroes in $K$ of any non-zcro polynomial in $K[X]$ with at most $k+1$ non-zero terms If $K$ can be embedded in the ficld $\mathbf{R}$ of real numbers, then $2 k$ is such a bound, by Descartes's rule of signs (sec [10, Section 109]), in particular, one can take $A(1, k, 1)=2 k$ My proof in the general case, which is given in Section 5 , invokes the following non-archimedean analogue of Descartes's rule of signs For a prime number $p$, let $\mathbf{Q}_{p}$ denote the field of $p$-adic numbers

Theorem 2. For any positive integer $k$ and any field $L$ that is a finte extension of $\mathbf{Q}_{p}$, for some prime number $p$, there exasts a posative anteger $B=B(k, L)$ with the following property Let $f \in L[X]$ be a non-zero polynomial with at most $k+1$ non-zero terms and with $f(0) \neq 0$ Then $f$ has at most $B$ zeroes in $L$, counted with multipliculies

B Poonen [7] has shown that this result can be extended to fields of Laurent series over finite ficlds if the zeroes are not counted with multiplicities I do not know whether there exist generalizations to systems of cquations in several vaulables, as un $[3]$

The proof of Theorem 2 is given in Section 4 It depends on a result that is even valid for algebraically closed fields Let an exponential valuaison on a ficld be defined as in [11, Section 1-3]

Theorem 3. For every prime number $p$, evcry posituve integer $k$, and cucry posative real number $r$ there exists a positive integer $C=C(p, k, r)$ with the follow ing property Lel $E$ be a field of characterstuc zero with an rxponc ntalul valuation $\nu E \rightarrow \mathbf{R} \cup\{\infty\}$ satisfying $\nu(p)=1$, and let $f \in E[X]$ be a non-zcro polynomial with at most $k+1$ non-zero terms Then $f$ has at most $C$ zeroes $x \in E$ with $\nu(x-1) \geq r$, counted with multiplucities

The theorem is reminiscent of the following obscrvation of Hajós (sce $[2,6$, Lemmd 1]) if $E$ is a trold of characteristic zero, and $f \in E[X]$ is a non-sero polynomial with at mosi $k+1$ non-zoro terms, then no non-vanishung zero of $f$ has multiplicity greater than $k$ My proof of Theorem 3, which is given in Section 3, may be viewed as a refinement of Hajos's argument It makes use of a property of binomial coefficients that is proved in Section 2

Hajós's result easily implies a result analogous to Thcorcm 3 for ficlds with an exponential valuation that have a residue class ficld of characteristic zcro, in this case one can take $C=k$, and the condition $\nu(x-1) \geq r$ can simply be replaced by $\nu(x-1)>0$ In the case of Theorem 3, polynomials like $X^{p^{N}}-1$ show that 
the bound $C$ necessarily depends on $r$. I do not know a valid variant of Theorem 3 that applies to algebraically closed fields of non-zero characteristic.

In Section 6 we extend, by a specialization argument, Theorem 1 to a more general class of ficlds and to polynomials in several variables.

Explicit values for $A, B$, and $C$ are given in Propositions 8.1, 7.2, and 7.1, respcctivcly. They satisfy

$$
\begin{aligned}
A(m, k, d) & =O\left(k^{2} \cdot 2^{m d} \cdot m d \cdot \log (2 m d k)\right), \\
B(k, L) & =O\left(k^{2} \cdot p^{f_{L}} \cdot e_{L} \cdot \log \left(2 e_{L} k\right)\right) \\
C(p, k, r) & =O\left(k+\frac{k \cdot \log (k /(r \log p))}{r \log p}\right)
\end{aligned}
$$

where $e_{L}$, and $f_{L}$ denote the ramification index and the residuc class ficld degrec of $L$ over $\mathbf{Q}_{\mu}$, respectively, and where the $O$-constants are absolute. These estimates give a fair impression of the order of magnitude of the best bounds that may be obtaincd by my method, for many values of the arguments; at the same time, my bounds are certainly open to numerical improvement.

From Theorem 1 and the value for $A$ just given one can deduce a lower bound for the largest degree of an irreducible factor of $f$, and an upper bound for the number of irreducible factors of $f$. These bounds depend only on $k$, on the degree $[K: \mathbf{Q}]$ of $K$, and on the degree $n$ of $f$. They are quite weak; in fact, for fixed $k$ and $[K: \mathbf{Q}]$ thcy are roughly proportional to $\log n$ and $n / \log n$, respectively. On the other hand, they are completely independent of the coefficients of $f$ and the discriminant of $K$.

It is an interesting problem to establish lower bounds for any values of $A$, $B$, and $C$ that make the conclusions of the theorems valid. Is the best value for $B(k, L)$ computable from $k$ and reasonable data - such as a defining polynomialspecifying $L$ ? It is not hard to show that the answer is affirmative if $k=1$. For the rest, I have not attempted to go beyond the case $k=2$ and $L=\mathbf{Q}_{2}$, which is treated in Section 9; it turns out that the argest number of non-vanishing zeroes that a "trinomial" $f \in \mathbf{Q}_{2}[X]$ can have in $\mathbf{Q}_{2}$ equals 6 (sce Proposition 9.2).

Cucker, Koiran, and Smale [1] cxhibited a polynomial time algorithm that computes all integer zeroes of a sparecly encoded polynomial $f \in \mathbf{Z}[X]$, where $\mathrm{Z}$ denotes the ring of integers. The vresent paper was originally inspired by one of the problems that they raise, namely that of computing the ratronal zoroes of $f$ in polynomial time as well. This can indeed be done, and in fact there is a polynomial time algorithm that determines all low degree irreducible factors of a sparsely cncoded polynomial in one variable with coefficients in an algebraic number field. This result is obtained in [5], by means of techniques different from those employed here.

Whenever, in the remainder of this paper, zeroes of a polynomial are counted, then it is understood that they are counted with multiplicitios. If $p$ is a prime

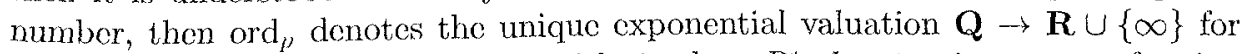
which $\operatorname{ord}_{p} p=1$. If $R$ is a ring with 1 , then $R^{*}$ denotes its group of units. 
If $n$ is a non-negative integer, and $t$ belongs to some $\mathbf{Q}$-algebra, then we write $\left(\begin{array}{l}t \\ n\end{array}\right)=\prod_{t=0}^{n-1} \frac{l-\imath}{n-\imath}$; this cquals 1 if $n=0$.

\section{Interpolating binomial coefficients}

For two non-negative integers $k$ and $n$, define $d_{k}(n)$ to be the least common multiple of all integers that can be written as the product of at most $k$ pairwise distinct positive integers that are at most $n$. Taking empty products to be 1 , we have $d_{k}(n)=1$ if $k=0$ or $n=0$. Clearly, $d_{k}(n)$ divides $n$ !, with equality if $n \leq k$. (In fact, it is not hard to show that one has $d_{k}(n)=n$ ! if and only if $n \leq 2 k+1$, a result that will not bo needed.) We have

$$
m \cdot d_{k-1}(m-1) \text { divides } d_{k}(n) \quad \text { if } 1 \leq m \leq n, k \geq 1 \text {. }
$$

This is immediate from the definition.

Proposition 2.2. Let $k$ and $n$ be non-negative integers, and let $T \subset \mathbf{Z}$ be a set of cardinaluty $k+1$. Then there exists a polynomial $h \in \mathbf{Z}[X]$ such that for each $t \in T$ one has $h(t)=d_{k}(n) \cdot\left(\begin{array}{l}t \\ n\end{array}\right)$.

Remark. With $d_{k}(n)$ replaced by $n !$, the conclusion of the proposition is trivial. This trivial result is strong enough to imply Theorem 3 in the case that $r>1 /(p-1)$, which suffices for the proofs of Theorems 2 and 1 .

Proof. We proceed by induction on $k$. If $k=0$ then $T=\{t\}$ for some integer $t$, and the constant polynomial $h=\left(\begin{array}{l}l \\ n\end{array}\right)$ has the required property, since $d_{0}(n)=1$. Next let $k>0$. Let an element $u \in T$ be chosen. The formal identity $(1+X)^{t}=$ $(1+X)^{u} \cdot(1+X)^{t-u}$ shows that for each $t \in \mathbf{Z}$ we have

$$
\left(\begin{array}{l}
t \\
n
\end{array}\right)=\sum_{m=0}^{n}\left(\begin{array}{c}
u \\
n-m
\end{array}\right)\left(\begin{array}{c}
t-u \\
m
\end{array}\right) \text {. }
$$

Using that $\left(\begin{array}{c}l-u \\ m\end{array}\right)=\frac{t-u}{m} \cdot\left(\begin{array}{c}t-u-1 \\ m-1\end{array}\right)$ for $m>0$, we obtain

$$
\left(\begin{array}{l}
t \\
n
\end{array}\right)=\left(\begin{array}{l}
u \\
n
\end{array}\right)+(t-u) \cdot \sum_{m=1}^{n} \frac{1}{m}\left(\begin{array}{c}
u \\
n-m
\end{array}\right)\left(\begin{array}{c}
t-u-1 \\
m-1
\end{array}\right) .
$$

Applying the induction hypothesis with $k-1, m-1$, and $\{t-u-1: t \in T, t \neq u\}$ in the roles of $k, n$, and $T$, respectively, we find that for each $m \in\{1,2, \ldots, n\}$ there cxists $h_{m} \in \mathbf{Z}[X]$ such that for each $t \in T, t \neq u$, one has $\left(\begin{array}{c}t-u-1 \\ m-1\end{array}\right)=$ $h_{m}(t-u-1) / d_{k-1}(m-1)$. Thercfore we have

$$
\left(\begin{array}{l}
t \\
n
\end{array}\right)=\left(\begin{array}{l}
u \\
n
\end{array}\right)+(t-u) \cdot \sum_{m=1}^{n} \frac{1}{m \cdot d_{k-1}(m-1)}\left(\begin{array}{c}
u \\
n-m
\end{array}\right) h_{m}(t-u-1)
$$


for each $t \in T$; this time we can include $t=u$, because of the factor $t-u$. Multiplying by $d_{k}(n)$ we obtain $d_{k}(n) \cdot\left(\begin{array}{l}l \\ n\end{array}\right)=h(t)$ for each $t \in T$, where

$$
h=d_{k}(n) \cdot\left(\begin{array}{l}
u \\
n
\end{array}\right)+(X-u) \cdot \sum_{m=1}^{n} \frac{d_{h}(n)}{m \cdot d_{h-1}(m-1)}\left(\begin{array}{c}
u \\
n-m
\end{array}\right) h_{m}(X-u-1) .
$$

By (2.1), the polynomial $h$ belongs to $\mathbf{Z}[X]$. This proves 2.2 .

Corollary 2.3. Let $k$ and $n$ be non-negative integers with $n>k$, and let $T \subset \mathbf{Z}$ be a set of cardinality $k+1$. Then there exist rational numbers $c_{0}, c_{1}, \ldots, c_{k}$ such that for each $i$ the denominator of $c_{t}$ divides $d_{k}(n) / i$ ! and such that for each $t \in T$ one has $\left(\begin{array}{l}l \\ n\end{array}\right)=\sum_{l=0}^{k} c_{\imath}\left(\begin{array}{l}t \\ l\end{array}\right)$.

Notc that $d_{k}(n) / i$ ! is actually an integer, for $0 \leq i \leq k<n$.

Proof. Let $h$ be as in Proposition 2.2. Rcplacing $h$ by its remainder upon division by $\prod_{t \in T}(X-t)$, wo may assume that $\operatorname{deg} h \leq k$. (In fact, if $h$ has been recursively constructed as in the proof of 2.2 , then it already satisfics this condition.) Since $i !\left(\begin{array}{l}X \\ \imath\end{array}\right)$ is an ith degrce polynomial in $\mathbf{Z}[X]$ with leading coefficient 1 , for each $\imath \geq 0$, we can write $h=\sum_{l=0}^{h} l_{\imath} ! !\left(\begin{array}{l}X \\ ,\end{array}\right)$ with $l_{t} \in \mathbf{Z}$. Now the numbers $c_{\imath}=l_{\imath} i ! / d_{k}(n)$ have the required properties. This proves 2.3 .

Proposition 2.4. Let $p$ be a prime number, and let $k, n$ be integers with $k \geq 0$ and $n \geq 1$. Then we have

$$
\operatorname{ord}_{p} d_{k}(n) \leq k \cdot\left[\frac{\log n}{\log p}\right],
$$

where $[x]$ denotes the largest integer not exceeding $x$.

Proof. From the definition of $d_{h}(n)$ one sees that the largest power of $p$ dividing $d_{k}(n)$ divides some product of at most $k$ positive integers that are at most $n$. Each of these integers has at most $[\log n / \log p]$ factors of $p$, so their product has at most $k \cdot[\log n / \log p]$ factors of $p$. This proves 2.4 .

Algorithm. Let $p$ be a prime nuriber, and let $k$ and $n$ be non-negative integers. To compute $\operatorname{ord}_{p} d_{k}(n)$, one detcrmines the least non-negative integer $j$ for which $\left[n / p^{1+1}\right] \leq k$; then one has

$$
\operatorname{ord}_{\gamma}, d_{h}(n)=j k+\operatorname{ord}_{p}\left(\left[n / p^{3}\right] !\right) .
$$

This computation is convenicntly carried out in base $p$; then one obtains $\left[n / p^{\prime}\right]$ by deleting the $p$-adically most significant $j$ digits of $n$, and if $s$ denotes the sum of the remaining digits then one has ord $p_{p}\left(\left[n / p^{3}\right] !\right)=\left(\left[n / p^{\prime}\right]-s\right) /(p-1)$. The elementary correctness proof of this method is left to the reader. 
For example, with $p=2, k=25, n=181$ one has in base 2

$$
\begin{gathered}
k=11001, \quad n=10110101, \quad \jmath=10(=p), \quad\left[n / p^{J}\right]=101101, \quad s=100, \\
\operatorname{ord}_{p}\left(\left[n / p^{\prime}\right] 1\right)=101001, \quad \operatorname{ord}_{p} d_{k}(n)=10 \quad 11001+101001=1011011,
\end{gathered}
$$

and the conclusion is that ord $d_{25}(181)=91$

\section{Zeroes close to 1}

We prove Theorem 3 For $p, k$, and $r$ as in the statement of the theorem, we define

$$
C(p, k, r)=\max \left\{m \geq 0 \quad m r-\operatorname{ord}_{p} d_{h}(m) \leq \max \left\{\imath r-\operatorname{ord}_{p}\left(\imath^{\prime}\right) \quad 0 \leq \imath \leq h\right\}\right\},
$$

with $d_{f}(m)$ as defined in Section 1 If $p, k$, and $r$ are fixed, then $\max \left\{\imath r-\operatorname{ord}_{p}\left(\iota^{\prime}\right)\right.$ $0 \leq \imath \leq k\}$ is constant, and $m r-\operatorname{ord}_{p} d_{k}(m)$ tends to infinity with $m$, this follows from 24 and the hypothess that $r>0$ Therefore $C(p, k, 1)$ is well-defined, and we have $C(p, k, r) \geq h$ since $d_{k}(k)=k^{\prime}$

We shall, with $p, k$, and $r$ as above, prove that $C=C(p, k, r)$ satusfies the conclusion of the theorem To do this, let $E, \nu$, and $f$ be as in the theorcm Replacing $E$ by an algebraic closure and cxtending $\nu^{\prime}$ we may, without loss of gencrality, assume that $E$ is algebrac ally closed

Write $f=\sum_{l \in I} a_{1} X^{\prime}$, where $T 15$ a set consisting of $k+1$ non-negative integers, and $a_{l} \in E$ for $t \in T$ Defino $g \in E[X]$ and $b, \in E$, for $\imath \geq 0$, by

$$
g=f(1+X)=\sum_{i \geq 0} b_{1} X^{c}
$$

Then we have

$$
b_{1}=\sum_{l \subset 7} a_{l}\left(\begin{array}{l}
l \\
l
\end{array}\right) \quad \text { for } \imath \geq 0
$$

Since $f \neq 0$ we have $g \neq 0$, so not all $b$, vanush

Denote by $n$ the number of seroes $x$ of $f$ in $E$ satusfying $\nu(x-1)>r$ This is the same as the number of zeroes $y$ of $g$ in $E$ satistying $\nu(y) \geq r$ Since $E$ is algebraically closed, that number can, by the theory of Newton polygons (see [11, Section 3-1]), be expressed in terms of $r$ and the valuations of the coefficienis $b_{\ell}$ of $g$, as follows

$$
n=\max \left\{m \geq 0 \quad \nu\left(b_{m}\right)+m s=\min \left\{\nu\left(b_{1}\right)+u r \quad, \geq 0\right\}\right\}
$$

It follows that we have

$$
\nu\left(b_{n}\right)+n r \leq \nu\left(b_{\imath}\right)+\iota r \quad \text { for all } \iota \geq 0
$$

Since not all $b_{l}$ vanush, this implies that $\nu\left(b_{n}\right) \neq \infty$

If $n \leq k$, then we have $n \leq C$, as required Suppose next that $n>k$ By 23 , there are rational numbers $c_{0}, c_{1}, \quad, c_{h}$, with the denominator of $c$, dividing 
$d_{k}(n) / \imath !$, such that for cach $t \in T$ one has

$$
\left(\begin{array}{l}
t \\
n
\end{array}\right)=\sum_{i=0}^{h} c_{\imath}\left(\begin{array}{l}
t \\
i
\end{array}\right) .
$$

Multiplying by $a_{t}$ and summing over $t \in T$ we find that

$$
b_{n}=\sum_{i=0}^{h} c_{i} b_{i} \text {. }
$$

Therefore we have

$$
\nu\left(b_{n}\right) \geq \min \left\{\nu\left(c_{l}\right)+\nu\left(b_{l}\right): 0 \leq i \leq k\right\} .
$$

The bound on the denominator of $c_{\text {, }}$ and the normalization $\nu(p)=1$ imply that $\nu\left(c_{2}\right) \geq \operatorname{ord}_{p}(n !)-\operatorname{ord}_{p} d_{h}(n)$. Also, we have $\nu\left(b_{2}\right) \geq \nu\left(b_{n}\right)+n r-2 r$. Therefore we find that

$$
\nu\left(b_{n}\right) \geq \min \left\{\operatorname{ord}_{p}(i !)-\operatorname{ord}_{p} d_{k}(n)+\nu\left(b_{n}\right)+n r-i r: 0 \leq i \leq k\right\} .
$$

Since $\nu\left(b_{n}\right) \neq \infty$, this implies that

$$
n r-\operatorname{ord}_{p} d_{h}(n) \leq \max \left\{i r-\operatorname{ord}_{p}(i !): 0 \leq i \leq k\right\} .
$$

Therefore we have $n \leq C$, as required. This proves Theorem 3 .

Remark. If $d_{k}(n)$ is replaced by $n$ ! in this proof (cf. the Rcmark in Section 2), thon it is still valid for $r>1 /(p-1)$, but not for $r \leq 1 /(p-1)$. This follows from $\operatorname{ord}_{p}(n !)=n /(p-1)+o(n)$ for $n \rightarrow \infty$.

\section{Local fields}

We prove Theorem 2. Let $L$ be as in the throrem. Then $L$ has a discrete valuation $\nu$ with a finite residue class ficld. Let $\nu$ be normalized such that $\nu(p)=1$ for some prime number $p$, and let $e$ be the unique positive integer for which $\nu\left(L^{*}\right)=\frac{1}{\mathbf{Z}} \mathbf{Z}$. We write $\mathcal{O}$ for the valuation $\operatorname{ring}\{x \in L: \nu(x) \geq 0\}$, and $P$ for the maximal idcal $\{x \in L: \nu(x)>0\}=\{x \in L: \nu(x) \geq 1 / c\}$ of $\mathcal{O}$. We denote by $q$ the cardinality of the finite residue class ficld $\mathcal{O} / P$. Let $C=C(p, k, 1 / e)$ be as in Theorem 3 . We shall show that $B=k \cdot(q-1) \cdot C$ satisfics the conclusion of Theorem 2 .

Let $f \in L[X]$ bo any non-zero polynomial with at most $k+1$ non-zero terms. Theorem 3 implies that $f$ las at most $C$ zcrocs in $1+P$. Applying this result to $f(u X)$, for $u \in \mathcal{O}^{*}$, we sec that $f$ has at most $C$ zcrocs in any $\operatorname{coset} u+P \in(\mathcal{O} / P)^{*}$. Sunming this over the $q-1$ elements of $(\mathcal{O} / P)^{\sharp}$, we derive that $f$ has at most $(q-1) \cdot C$ zeroes in $\mathcal{O}^{*}$. Applying this result to $f(a X)$, for $a \in L^{*}$, we find that $f$ has at most $(q-1) \cdot C$ zorocs in any $\operatorname{coset} a \mathcal{O}^{*} \in L^{*} / \mathcal{O}^{*}$; or, cquivalently, that $f$ has at most $(q-1) \cdot C$ zeroes $x \in L^{+}$for which $\nu(x)$ assumes a given finite value. Since by the theory of Newton polygons we have $\#\left\{\nu(x): x \in L^{*}, f(x)=0\right\} \leq k$ (see 
also [8, Lemma 2 1]), we can now conclude that $f$ has at most $k(q-1) C$ zeroes in $L^{*}$ If we restrict, as in Theorem 2 , to polynomials with $f(0) \neq 0$, then this is also an upper bound for the number of zcroes of $f$ in $L$ This proves Theorem 2

Remark. If the conclusion of Theorem 3 is avalable only for $r>1 /(p-1)$ (cf the Remark in Section 3), then the preceding proof still works if one replaces the cosets $u+P \in(\mathcal{O} / P)^{*}$ by $u+P^{l} \in\left(\mathcal{O} / P^{l}\right)^{*}$, where $l / c>1 /(p-1)$, then the factor $q-1$ needs to be replaced by the order $(q-1) q^{l-1}$ of $\left(\mathcal{O} / P^{l}\right)^{+}$, and the conclusion is that one can take $B(k, L)=k(q-1) \quad q^{l-1} C(p, k, l / e)$

\section{Number fields}

We prove Theorem 1 Let $m, k$, and $d$ bc as in Theorem 1 Let $p$ be any prime number, for example $p=2$, and let $\overline{\mathbf{Q}}_{p}$ be an algebraic closurc of $\mathbf{Q}_{p}$ By [4 Chap II, Prop 14], the field $\mathbf{Q}_{p}$ has only fintely many extensions of degree at most $d m$ in $\mathbf{Q}_{p}$. Let $L$ be the composite of all these cxtensions, it is of finite degice over $\mathbf{Q}_{p}$, We shall show that $A=B(k, L)$ satisfies the conclusion of the theorem

Let $K, f$, and $g$ be as in Theorem 1 We may embed $K$ as a subfield in $\overline{\mathbf{Q}}_{p}$ Then $K \quad \mathbf{Q}_{p}$ has degree at most $m$ over $\mathbf{Q}_{p}$. Hence any zcro of $f$ in $\overline{\mathbf{Q}}_{p}$ that has degree at most $d$ over $K$ lies in an extension of degree at most $d m$ of $\mathbf{Q}_{p}$, and therefore in $L$ Thus, the number of zeroes of $f$ in $\mathbf{Q}_{p}^{+}$that have degree at most $d$ over $K$ is hounded by $B(k, L)$ This imples that the degrec of $g$ is at most $B(k, L)$, as required This proves Theorem 1

\section{A generalization}

For a ring $R$ and a positive integer $n$, we denote by $R\left[X_{1}, \quad, X_{n}\right]$ the polynomal ring in $n$ variables $X_{1}, \quad, X_{n}$ over $R$ A polynomial in one variable is called monic if it has leading coefficent 1

Proposition 6.1. Let $m, k, d$ bc positive integers, and let $A=A(m, k, d)$ be any positive integer for which the conclusion of Theorem 1 is true Suppose that $K$ is a ficld that 29 of degree at most $m$ over a purcly transcendental field extension $K_{0}$ of $\mathrm{Q}$, that $n$ us a positve unteger, and that $f \in K\left[X_{1}, \quad, X_{n}\right]$ is a non-zero polynomial with at most $k+1$ terms $L c t g \in K\left[X_{1}, \quad, X_{n}\right]$ be a factor of $f$ such that for parh $\imath \in\{1,2, \quad, n\}$, every arredur uble factor of $g$ has degree at most $d$ in $X_{\iota}$, and $g$ is not divisible by $X$, Then, for each $\imath \in\{1,2, \quad, n\}$, the degree of $g$ in $X_{l}$ is at most $A$

Proof We know the result to be true of $K_{0}=\mathrm{Q}$ and $n=1$ We first extend this to the case $K_{0}=\mathrm{Q}(t \quad t \in T)$ for some collection $T$ that is algebralcally 
independent over $\mathbf{Q}$, still for $n=1$ Let $K_{0}$ be such, let $K=K_{0}(u)$ be of degree $l$ over $K_{0}$, and let $f, g \in K[X]$ be as in the statement of 61 Without loss of generality we assume that $f$ and $g$ are monic Let $R_{0} \subset K_{0}$ be a subring of the

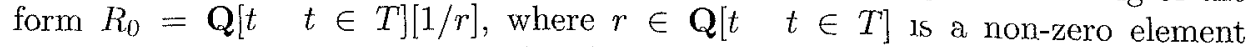
that is chosen in such a manner that $R_{0}$ contains the coefficients of the following elements of $K$, when expressed on the $K_{0}$-basis $\left(u^{2}\right)_{i=0}^{l} \quad 1$ of $K$ the coefficients of $f$, the coefficients of the monic urieducible factors of $g$, the inverse of $g(0)$, and $u^{l}$ Then $R=\sum_{l=0}^{l-1} R_{0} \quad u^{l}$ is a subring of $K$ that is isomorphic to $R_{0}[U] /(h)$ for some monic polynomial $h=\sum_{l=0}^{l} h_{2} U^{\prime} \in R_{0}[U]$, and one has $f, g \in R[X]$ Next, one chooses rational numbers $a_{t}$, for $t \in T$, such that $\left(a_{\ell}\right)_{t \in \Gamma}$ is not a zero of $r$, and one defines $\varphi \quad R_{0} \rightarrow \mathbf{Q}$ by substituting $a_{t}$ for $t$ Adjoining a zero of $\sum_{\imath} \varphi\left(h_{\imath}\right) U^{\imath}$, one can extend $\varphi$ to a ring homomorphism from $R$ to some algebraic number field $K_{1}$ of degree at most $l$ over $\mathbf{Q}$ The induced map $R[X] \rightarrow K_{1}[X]$ sending $X$ to $X$ maps $f$ to a monic polynomial $f_{1} \in K_{1}[X]$ with at most $k+1$ non-zero terms, and $g$ to a factor $g_{1}$ of $f_{1}$ that has the same degree as $q$, that can be witten as the product of polynomials of degree at most $d$, and that satusfies $g_{1}(0) \neq 0$ Hence by what we know about $K_{1}$, the degree of $g$ is at most $A$ This proves the case $n=1$ of 61

For general $n$, let the notation again be as in 61 , and let $\imath \in\{1,2, \quad, n\}$ View $f$ and $g$ as polynomials in a single variable $X_{\iota}$ with coefficients in the field $K(X, j \neq \imath)$ of fractions of the polynomial ring in the remaining variables, this field is of degree at most $m$ over the field $K_{0}(X, \mid \jmath \neq \imath)$, which is purely transcendental over $\mathbf{Q} \operatorname{In} K(X, \mid) \neq \imath)\left[X_{2}\right]$, the polynomial $g$ is a product of polynomials of degree at most $d$, and it is not divisible by $X_{\iota}$ Hence by what we know about the case $n=1$, the degree of $g$ is at most $A$ This proves 61

\section{Explicit bounds: the local case}

Proposition 7.1. Let $C(p, k, r)$ be as defuned in Section 3, and write

$$
c=\frac{\exp 1}{(\exp 1)-1}, \quad v=\max \left\{\imath-\left(\operatorname{ord}_{p}\left(\imath^{\prime}\right)\right) / r \quad 0 \leq \imath \leq k\right\}, \quad w=\frac{k}{r \log p}
$$

Then we have

$$
C(p, k, r) \leq c \quad(v+w \log w) \leq c \quad k \quad\left(1+\frac{\log (k /(r \log p))}{r \log p}\right)
$$

We note that $c=158197671$

Proof The last inequality follows from the fact that $v \leq k$ We prove the first inequality By the definition of $C(p, k, r)$, it suffices to show that

$$
m-\frac{\operatorname{ord}_{p} d_{\ell}(m)}{r}>v \quad \text { for all } m>c(v+w \log w)
$$


The function $1-(\log x) / x$ of a positive variable $x$ assumes 1 ts minimum $1 / c$ at $x=\exp 1$ Hence for all $x>0$ we have $x \geq(\log x)+x / c$ Now let $m$ be an integer, $m>c(v+w \log w)$, we have $m>1$, since $v \geq 1$ and $w \log w \geq-\exp (-1)$ Taking $x=m / w$ and applying 24 we find that

$$
\begin{gathered}
m=w \quad x \geq w \log x+\frac{w x}{c}=w \log m-w \log w+\frac{m}{r} \\
>w \log m+v=\frac{k \log m}{r \log p}+v \geq \frac{\operatorname{ord}_{p} d_{k}(m)}{r}+v,
\end{gathered}
$$

as required This proves 71

Let $p$ be a prime number, and let $L$ be a finte field extension of $\mathbf{Q}_{p}$ Denote by $e_{L}$ and $f_{L}$ the ramification index and the residue class field degree of $L$ over $\mathbf{Q}_{p}$, respectively For a positive integer $k$ we define

$$
B(k, L)=k \quad\left(p^{\int_{I}}-1\right) C\left(p, k, 1 / e_{L}\right),
$$

with $C\left(p, k, 1 / e_{L}\right)$ as defined in Section 3

Proposition 7.2. With $B(k, L)$ as just defined, the conclusion of Theorem 2 is valud Moreover, with $c$ as in 71 and $e_{J}$ and $f_{L}$ as just defined, we have

$$
B(k, L) \leq c k^{2}\left(p^{/ L}-1\right)\left(1+\frac{e_{L} \log \left(e_{L} k / \log p\right)}{\log p}\right)
$$

Proof This is clcar from Soction 4 and 71

Example: $k=1$. One can show that $C\left(p, 1,1 / \epsilon_{I}\right)=s_{I} e_{I}+1$, where $s_{I}=$ $\max \left\{s \in \mathbf{Z} \quad s \quad e_{I}+1 \geq p^{s}\right\}$, so one has $B(1, L)=\left(p^{f_{I}}-1\right)\left(s_{I} c_{I}+1\right)$ The smallest value for $B$ that makes the conclusion of Theorem 2 valid with $k=1$ is equal to the number of roots of unity in $L$, which is of the form $\left(p^{f_{1}}-1\right) p^{i /}$, where $r_{L}$ is a non-negative integer for which $(p-1) p^{r_{J}}{ }^{\prime}$ divides $e_{I}$

\section{Explicit bounds: the global case}

For positive integers $m, k$, and $d$, we define

$$
A(m, k, d)=k \sum_{\jmath=1}^{m d}\left(2^{\prime}-1\right) \quad C\left(2, k, \frac{1}{[m d / \jmath] m d}\right),
$$

where $[x]$ denotes the greatest integer not exceeding $x$, and the function $C$ is as defined in Section 3 
Proposition 8.1. With $A(m, k, d)$ as just defined, the conclusion of Theorem 1 is valid Moreover, we have

$$
A(m, k, d)<\frac{c}{\log 2} k^{2}(m d+10) 2^{m d+1} \log \left(\frac{k m d}{\log 2}\right),
$$

where $c$ is as in 71

We note that $c / \log 2=228230995$

The proof of 81 requires a more refined approach than the one taken in Section 5

We denote by $\overline{\mathbf{Q}}_{2}$ an algebraic closure of the field $\mathbf{Q}_{2}$ of 2-adic numbers, and by $\nu \quad \overline{\mathbf{Q}}_{2} \rightarrow \mathbf{Q} \cup\{\infty\}$ the extension of the natural exponential valuation on $\mathbf{Q}_{2}$, normalized so that $\nu(2)=1$ We fix a group homomorphism $\mathbf{Q} \rightarrow \mathbf{Q}_{2}^{*}$, written $r \mapsto 2^{\gamma}$, with the property that $2^{\perp}=2$, to construct such a group homomorphism, one chooses inductively $2^{1 / n^{\prime}}$ to be an $n$th root of $2^{1 /(n-1)^{\prime}}$, and one defines $2^{a / n^{i}}$ to be the ath power of $2^{1 / n^{\prime}}$, for $a \in \mathbf{Z}$ We have $\nu\left(2^{r}\right)=r$ for each $r \in \mathbf{Q}$ For positive integers, and $e$, we define the subgroups $U_{c}$ and $T$, of $\overline{\mathbf{Q}}_{2}^{*}$ by

$$
U_{\mathrm{s}}=\{a \quad \nu(x-1) \geq 1 / e\}, \quad T_{1}=\left\{\zeta \quad \zeta^{2^{\prime}}{ }^{1}=1\right\}
$$

We have $U_{c} \subset U_{\mathrm{c}^{\prime}}$ if $e \leq e^{\prime}$, and $T, \subset T, 11 \rho$ divides $\gamma^{\prime}$

Lemma 8.2. Let $k, j$, and e be positıve integers, and let $f \in \overline{\mathbf{Q}}_{2}[X]$ be a non-zero polynomial having $k+1$ non-zero terms Then $f$ has at most $k\left(2^{7}-1\right) C(2, k, 1 / e)$ zeroes in the subgroup $2^{\mathbf{Q}} T_{j} U_{c}$ of $\mathbf{Q}_{2}^{+}$

Proof This is done by a straightforward extension of the argument of Section 4 one knows from Theorem 3 that $f$ has at most $C(2, k, 1 / e)$ zeroes in $U_{r}$, and one deduces that the same is true for any coset of $U_{t}$, next one observes that $T_{3}$ has order $2^{\prime}-1$, and one derives that $f$ has at most $\left(2^{\prime}-1\right) \quad C(2, k, 1 / e)$ zeroes in each coset $2^{r} T, U_{\text {c }}$ of $T_{3} U_{t}$, and one concludes the proof using the fact that $\nu$ assumes at most $k$ different values $r$ at the seroes of $f$ in $\mathbf{Q}_{2}^{*}$ This proves 82

Lemma 8.3. Let $n$ be a positıve integer, and let $L$ be aro exlcnsion of $\mathbf{Q}_{2}$ of degree at most $n$ inside $\mathbf{Q}_{2}$ Then there exiscs an integer $\jmath \in\{1,2, \quad, n\}$ such that $L^{*} \subset 2^{\mathrm{Q}} T, U_{[n / 1] n}$

Proof Let $f_{I}$ and $e_{I}$ be as in Section 7 , and put $M=L\left(2^{1 / \kappa_{L}}\right)$ We clam that $\gamma=f_{M}$, the residue class field regrec of $M$ over $\mathbf{Q}_{2}$, has the stated properties To prove this, denote by $e^{\prime}$ and $f^{\prime}$ the ramification index and residue class field degrec of $M$ over $L$ Then we have $e^{\prime} f^{\prime}=\left[\begin{array}{ll}M & L\end{array}\right] \leq e_{L}$, and therefore

$$
f_{M} \leq c^{\prime} \quad f_{M}=e^{\prime} \quad f^{\prime} \quad f_{L} \leq e_{L} \quad f_{L}=\left[\begin{array}{ll}
L & \mathrm{Q}_{2}
\end{array}\right] \leq n
$$

This proves, first, that $y=f_{M}$ does bclong to $\{1,2, \quad, n\}$, and secondly, that $e^{\prime} \leq\left[n / f_{M}\right]=[n / \eta]$ Hence the ramification index $e_{M}$ of $M$ over $\mathbf{Q}_{2}$ satisfies $e_{M}=e^{\prime} \quad e_{I} \leq[n / 1] \quad n$ Therefore each $x \in M$ with $\nu(x-1)>0$ belongs to 
$U_{[n / j] n}$. From $j=f_{M}$ it follows that $T_{j} \subset M^{*}$, and that $T$, is in fact a system of representatives for the group of units of the residue class field of $M$ (see [9, Chap. 2, Prop. 8(iii)]]). It follows that the kernel of $\nu: M^{*} \rightarrow \mathbf{Q}$ is contained in $T_{\gamma} \cdot U_{[n / 1] n}$. Now, in order to prove that $L^{*} \subset 2^{\mathrm{Q}} \cdot T, U_{[n / \tau] n}$, let $x$ belong to $L^{*}$. Then $\nu(x)=r$ for some $r \in \frac{1}{\rho_{L}} \mathbf{Z}$, so the elcment $x \cdot 2^{-r}$, which does belong to $M^{*}$, is in the kernel of $\nu$. Therefore we have $x \in 2^{r} \cdot T_{\jmath} \cdot U_{[n / /] n} \subset 2^{\mathrm{Q}} \cdot T_{\jmath} \cdot U_{[n / J] n}$, as required. This proves 8.3 .

One can show that the integer $e^{\prime}$ occurring in the proof above is a power of 2 . This observation may be used to improve our value for $A(m, k, d)$, but it will not change its order of magnitude.

We turn to the proof of 8.1 . Let $m, k, d$ be positive integers, and let $K, f, g$ be as in Theorem 1 . We may assume that $K$ is a subfield of $\overline{\mathbf{Q}}_{2}$. Then every zero of $g$ in $\overline{\mathbf{Q}}_{2}$ lies in an extension of degree at most $n=m d$ of $\mathbf{Q}_{2}$, so by Lemma 8.3 also in $\bigcup_{j=1}^{n}\left(2^{\mathrm{Q}} \cdot T_{\jmath} \cdot U_{[n / \jmath] n}\right)$. From Lemma 8.2 it now follows that the number of zeroes of $g$ in $\overline{\mathbf{Q}}_{2}$ is at most

$$
\sum_{j=1}^{n} k \cdot\left(2^{\jmath}-1\right) \cdot C(2, k, 1 /([n / \jmath] n))=A(m, k, d) .
$$

Hence $A(m, k, d)$ is an upper bound for the degree of $g$. This proves the first assertion of 8.1. We prove the second assertion. From 7.1 we obtain

$$
A(m, k, d) \leq c \cdot k^{2} \cdot \sum_{j=1}^{n}\left(2^{\jmath}-1\right) \cdot\left(1+\frac{[n / j] n \cdot \log ([n / j] n k / \log 2)}{\log 2}\right)
$$

where we still write $n=m d$. For $[n / 2]<\jmath \leq n$ we have $[n / j]=1$, and for $1 \leq j \leq[n / 2]$ we have $[n / j] \leq n$ and $\log ([n / j] n k / \log 2) \leq 2 \log (n k / \log 2)$. This leads to

$$
\begin{gathered}
A(m, k, d)<c \cdot k^{2} \cdot\left(2^{n+1}+2^{n+1} \cdot \frac{n \cdot \log (n k / \log 2)}{\log 2}+2^{[n / 2]+1} \cdot \frac{n^{2} \cdot 2 \log (n k / \log 2)}{\log 2}\right) \\
\leq c \cdot k^{2} \cdot 2^{n+1} \cdot \frac{(n+10) \cdot \log (n k / \log 2)}{\log 2}
\end{gathered}
$$

the second inequality being obtained by a routine argument. This proves 8.1 .

\section{Two-adic trinomials}

In this section we determine bow many zeroes a polynomial of the form

$$
f=a+b X^{t}+c X^{u} \quad \text { with } a \in \mathbf{Q}_{2}^{*}, b, c \in \mathbf{Q}_{2}, t, u \in \mathbf{Z}, 0<t<u,
$$

may have in $\mathbf{Q}_{2}$; here we still count zeroes with their multiplicities. We let the function $C$ be as defined in Section 3 , and we write $\nu$ for the natural exponcntial valuation on $\mathbf{Q}_{2}$. 
According to the first assertion of 72 , with $k=2, p=2, L=\mathbf{Q}_{2}, e_{L}=1$, and $f_{L}=1$, an upper bound for the number of zeroes of any $f$ as in (91) in $\mathbf{Q}_{2}$ is given by $2 C(2,2,1)$, which by a durect computation is found to be 8 (The second assertion of 72 gives the upper bound 160018 ) The following result shows that the best upper bound is 6

\section{Proposition 9.2.}

(a) Let $f$ be as in (9 1) Then the number of zeroes of $f$ in $\mathbf{Q}_{2}$ equals $0,1,2,3$, 4 , or 6 , and if it equals 4 or 6 then $t$ and $u$ are both even

(b) For any $n \in\{0,1,2,3,4,6\}$ there exusts $f$ as in $(91)$, with $b \neq 0$ and $c \neq 0$, such that the number of zeroes of $f$ in $\mathbf{Q}_{2}$ equals $n$

In the proof we use a variant of 22 We write $\mathbf{Z}_{p}$ for the ring of $p$-adic integers

Proposition 9.3. Let $p$ be a prime number, $n$ a non-negatvve integer, and $T$ a finite non-empty subset of $\mathbf{Z}$ Write $T_{1}=\{t \in T \quad(t \bmod p)=\jmath\}$ for each $\jmath \in \mathbf{Z} / p \mathbf{Z}$, and put $k=\max \left\{\# T_{j}, \jmath \in \mathbf{Z} / p \mathbf{Z}\right\}-1$ Then there exists a polynomial $h \in \mathbf{Z}_{p}[X]$ such that for each $t \in T$ one has $h(t)=d_{k}(n) \quad\left(\begin{array}{l}l \\ n\end{array}\right)$

Proof Let $\jmath \in \mathbf{Z} / p \mathbf{Z}$ be such that $T_{\jmath}$ is non-empty, and put $k(\jmath)=\# T_{\jmath}-1$ Applying 22 to $T_{j}$, we obtain a polynomial $h_{j} \in \mathbf{Z}[X]$ with the property that for each $t \in T_{\text {, }}$ one has $h_{l}(t)=d_{h(\jmath)}(n)\left(\begin{array}{l}t \\ n\end{array}\right)$ Next define

$$
g_{J}=1-\prod_{l \in \mathcal{T}}\left(1-\prod_{u \in I} \frac{X-u}{v \notin 1,}\right)
$$

We have $g_{j} \in \mathbf{Z}_{p}[X]$, since none of the denominators $t-u$ is divisible by $p$ Also, we have $g_{j}(t)=1$ for $t \in T$, and $g_{j}(u)=0$ for $u \in T, u \notin T_{\text {, }}$

It is now straightforward to verify that the polynomial

$$
h=\sum_{\jmath} g_{3} h, d_{h}(\imath) / d_{h(\jmath)}(n)
$$

has the properties stated in 93 , note that for each $\jmath$ we have $d_{l}(n) / d_{h(\eta)}(n) \in \mathbf{Z}$, since $k(\jmath) \leq k$ This proves 93

Proof of 92 (a) Let $f$ be as in $(91)$ Let it first be assumed that $t$ or $u$ is odd, $m$ this case 92 (d) asserts that $f$ has at most 3 zeroes in $\mathbf{Q}_{2}$ To prove this, we observe that $T=\{0, t, u\}$ contams integers of both parities, so when we apply 93 we can lake $k=1$ (as opposed to $k=2$ when we apply 22 ) With this improvement, the argument given in Section 3 shows that the number of zeroes of $f$ in $\mathbf{Z}_{2}^{*}=1+2 \mathbf{Z}_{2}$ is at most $C(2,1,1)=2$ (as oplosed to $C(2,2,1)=4$ ) If $\nu$ assunies at most 1 value at the set of zeroes of $f$ in $\mathbf{Q}_{2}$, then the argument of Section 4 now implies that $f$ has at most 2 zeroes in $\mathbf{Q}_{2}$ Assume therefore that $\nu$ assumes at least 2 values at the zeroes of $f$ in $\mathbf{Q}_{2}$ Let $r$ and $s$ be zeroes of $f$ in $\mathbf{Q}_{2}$ with $\nu(r)>\nu(s)$ By the theory of Newton polygons, cach zero of $f$ in $\mathbf{Q}_{2}$ is in $r \quad \mathbf{Z}_{2}^{*}$ or in $s \mathbf{Z}_{2}^{*}$, and the 
polynomials $f(r X)$ and $f(s X)$ have the shape

$$
\begin{aligned}
& f(r X)=\left(a^{\prime}+b^{\prime} X^{\iota}+c^{\prime} X^{u}\right) \quad d^{\prime}, \quad \text { with } a^{\prime}, b^{\prime} \in \mathbf{Z}_{2}^{*}, c^{\prime} \in 2 \mathbf{Z}_{2}, d^{\prime} \in \mathbf{Q}_{2}^{+}, \\
& f(s X)=\left(a^{\prime \prime}+b^{\prime \prime} X^{t}+c^{\prime \prime} X^{u}\right) d^{\prime \prime}, \quad \text { with } a^{\prime \prime} \in 2 \mathbf{Z}_{2}, b^{\prime \prime}, c^{\prime \prime} \in \mathbf{Z}_{2}^{*}, d^{\prime \prime} \in \mathbf{Q}_{2}^{*}
\end{aligned}
$$

Each of these polynomials has 1 as a zero and has at most 2 zeroes in $\mathbf{Z}_{2}^{*}$ If $t$ is odd, then 1 is a simple zero of the reduction of $f(r X) / d^{\prime}$ modulo 2 , so by Hensel's lemma (see [11, Cor 2-2-6]) it is the unique zero of $f(r X)$ in $\mathbf{Z}_{2}^{*}=1+2 \mathbf{Z}_{2}$ If $t$ is even then $u$ is odd, and 1 is a simple zero of the reduction of $f(s X) / d^{\prime \prime}$ modulo 2 , so by Hensel's lemma it is the unique zero of $f(s X)$ in $\mathbf{Z}_{2}^{*}$ In either case, one of the two polynomials has a unique zero in $\mathbf{Z}_{2}^{*}$, and the other at most 2 Therefore $f$ has at most 3 zeroes in $\mathrm{Q}_{2}$, as asserted

Next assume that $t$ and $u$ are even We can write $t=t_{0} 2^{l}$ and $u=u_{0} 2^{l}$, where $l$ is a positive integer and $t_{0}$ or $u_{0}$ is odd Then we have $f=f_{0}\left(X^{2^{l}}\right)$, where $f_{0}=a+b X^{l_{0}}+c X^{u_{0}}$, and the zeroes of $f$ arc the $2^{l}$ th roots of the zeroes of $f_{0}$ By the above, $f_{0}$ has at most 3 zeroes in $\mathbf{Q}_{2}$, and since $\mathbf{Q}_{2}$ contains exactly 2 roots of unity, each of these zeroes that has a $2^{l}$ th root in $\mathbf{Q}_{2}$ has exactly 2 of them Hence the number of zeroes of $f$ in $\mathbf{Q}_{2}$ equals $0,2,4$, or 6 This proves (a)

(b) One easily verifies that the polynomials

$$
X^{2}+X+1, \quad X^{3}+X^{2}-2, \quad X^{2}-5 X+4, \quad X^{4}-5 X^{2}+4
$$

have exactly $0,1,2,4$ zeroes in $\mathbf{Q}_{2}$, respectively (They have in fact the same property over $\mathbf{Q}$ and $\mathbf{R}$ ) Next consider the polynomial

$$
f=3 X^{5}+X-4
$$

One has

$$
\frac{f(8 X+1)}{128}=768 X^{5}+480 X^{4}+120 X^{3}+15 X^{2}+X \equiv X \quad(X-1) \bmod 2
$$

By Hensel's lemma, $f(8 X+1)$ has two zerocs in $\mathbf{Z}_{2}$, so $f$ has two zeroes in $1+8 \mathbf{Z}_{2}$ Also, one has

$$
\frac{f(4 X)}{4}=3 \quad 2^{8} \quad X^{5}+X-1 \equiv X-1 \bmod 8,
$$

so $f(4 X)$ has a zero in $\mathbf{Z}_{2}$ that is $1 \bmod 8$, and $f$ has a zero in $2^{2}\left(1+8 \mathbf{Z}_{2}\right)$ This shows that $f$ has at least 3 zeroes in $\mathbf{Q}_{2}$, and by (a) it has no others Since each element of $1+8 \mathbf{Z}_{2}$ is a square in $\mathbf{Q}_{2}$, each of the 3 zcroes of $f$ has two square roots in $\mathbf{Q}_{2}$ Therefore the polynomial $3 X^{10}+X^{2}-4$ has exactly 6 zerocs in $\mathbf{Q}_{2}$ This proves 92

Remark. The arguments used in the proof of 92 lead to the following general result Let the hypotheses and the notation be as in 72 , and define

$$
B^{\prime}(k, L)=w_{L}\left(p^{/ t}-1\right)\left(1+(k-1) C\left(p, k-1,1 / e_{L}\right)\right),
$$

where $w_{I}$ denotes the number of roots of unity in $L$ that have $p$ power order Then the conclusion of Theorem 2 is vald with $B^{\prime}(k, L)$ in the place of $B(k, L)$ For $k=1$, we have $B^{\prime}(1, L)=\omega_{L}\left(p^{f_{1}}-1\right)$, which is the number of all roots of 
unity in $L$; it follows that for $k=1$ the bound $B^{\prime}(k, L)$ cannot be improved. If $k>1$, then one has $B^{\prime}(k, L)<B(k, L)$ for all $L$ with $w_{L}=1$; but if $w_{L}>1$, then one has $B^{\prime}(k, L)>B(k, L)$ for all $k$ exceeding a bound that depends on $L$.

\section{References}

[1] Cucker, F., Koiran, P., Smale, S., A polynomial time algorithm for diophantine equations in one variable. J. Symbolic Comput., to appear.

[2] Hajós, G., [Solution to problem 41] (in Hungarian). Mat. Lapok 4 (1953), 40-41.

[3] Khovanskiř, A.G., Fewnomials. Amer. Math. Soc., Providence 1991.

[4] Lang, S., Algebraic number theory. 2nd ed. Springer, New York 1994.

[5] Lenstra, H.W., Jr., Finding small degree factors of lacunary polynomials. This volume, 267-276.

[6] Montgomery, H.L., Schinzel, A., Some arithmetic properties of polynomials in several variables. In: Transcendence theory: advances and applications (ed. by A. Baker, D.W. Masser), Chapter 13, 195-203. Academic Press, London 1977.

[7] Poonen, B., Zeros of sparse polynomials over local fields of characteristic $p$. Math. Res. Lett., to appear.

[8] Schacher, M., Straus, E.G., Some applications of a non-Archimedean analogue of Descartes' rule of signs. Acta Arith. 25 (1974), 353-357.

[9] Serre, J-P., Corps locaux. Hermann, Paris 1962.

[10] Weber, H., Lehrbuch der Algebra, vol. I. 2nd ed. Vieweg, Braunschweig 1912.

[11] Weiss, E., Algebraic number theory. McGraw-Hill, New York 1963. 